\title{
Effect of Magnetic Fields on TiAu TES Bolometers for the SAFARI Instrument on the SPICA FIR Telescope
}

\author{
R.A. Hijmering • P. Khosropanah • M. Ridder • M. Lindeman • L. Gottardi • \\ M. Bruijn · J. van der Kuur • P. de Korte · J.-R. Gao • H. Hoevers • B. Jackson
}

Received: 29 July 2011 / Accepted: 5 January 2012 / Published online: 1 February 2012

(C) Springer Science+Business Media, LLC 2012

\begin{abstract}
At the Netherlands Institute for Space Research (SRON) we are developing Transition Edge Sensors (TES) bolometers for the SAFARI Imaging Spectrometer on the SPICA telescope. With the current design of the SPICA telescope a background magnetic field of about a Gauss with fluctuations of a couple percent is expected. The effect of this external magnetic field on the bolometer needs to be well understood in order to design effective shielding. We present the results of an investigation of magnetic field on the performance of TES bolometers. For this experiment we used a Ti/Au TES bolometer which has a measured NEP of $5.0 \times 10^{-19} \mathrm{~W} / \mathrm{Hz}^{1 / 2}$. The TES is exposed to a parallel and perpendicular magnetic field of varying strength and the baseline and responsivity are determined. We see that the bolometers are sensitive to the perpendicular field but are relative insensitive to the parallel magnetic field. Fluctuations with a period of $30 \mathrm{mG}$ are present as function of perpendicular magnetic field.
\end{abstract}

Keywords Bolometer · Transit Edge Sensor · Far Infrared · Infra Red · SPICA · SAFARI

\section{Introduction}

SPICA [1] is a background limited infrared mission, scheduled to be launched in 2018 by JAXA. The instruments in the focal plane will be: MIR Coronagraph, MIR

\footnotetext{
R.A. Hijmering $(\bowtie) \cdot$ P. Khosropanah $\cdot$ M. Ridder · M. Lindeman · L. Gottardi · M. Bruijn ·

J. van der Kuur · P. de Korte $\cdot$ J.-R. Gao $\cdot$ H. Hoevers $\cdot$ B. Jackson

SRON Netherlands Institute for Space Research, Sorbonnelaan 2, 3584 CA Utrecht, The Netherlands

e-mail: r.hijmering@sron.nl
}

J.-R. Gao

Kavli Institute of NanoScience, Delft University of Technology, Delft, The Netherlands 

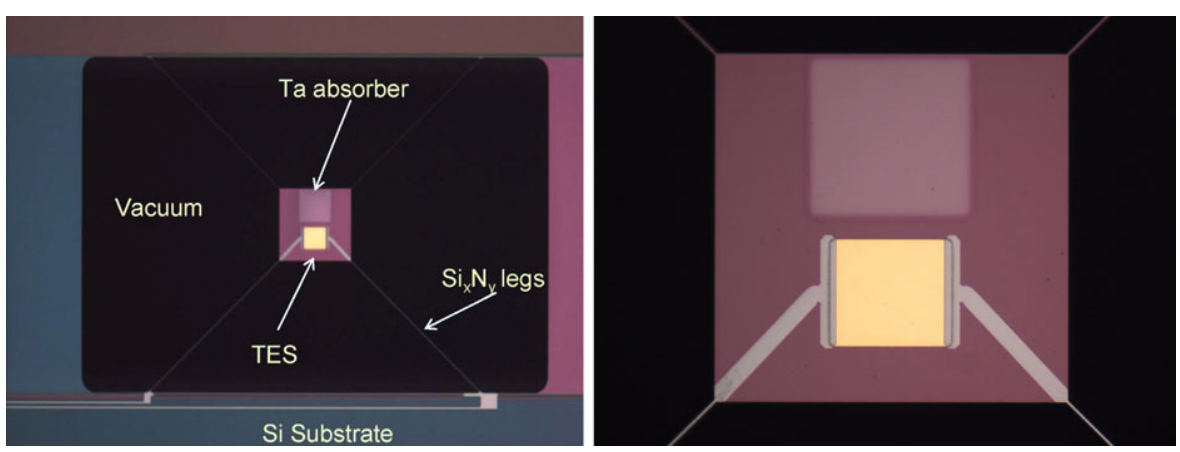

Fig. 1 (Color online) (Left) Image of a Ti/Au TES and a thin Ta absorber on a SiN supporting island. (Right) a zoom of the TES and the absorber on the $\mathrm{SiN}$ island

Camera and Spectrometer and the SAFARI imaging spectrometer [2], the European contribution. SAFARI will contain three imaging arrays of TES bolometers [3] which will operate in the bands: $35-60 \mu \mathrm{m}, 60-110 \mu \mathrm{m}$ and $110-210 \mu \mathrm{m}$. Next to its imaging capabilities SAFARI will also be used to obtain spectral information using a Fourier Transform Spectrometer.

It is known that superconducting materials are sensitive to magnetic fields. In order to design sufficient shielding against the background magnetic field in the limited space available the effect of magnetic field on the TES bolometers needs to be well understood. Several groups have reported on the influence of magnetic field on TES detectors. Ullom et al. [5] showed that applying a perpendicular magnetic field suppresses the excess noise in X-ray MoCu TES detectors by $\alpha$ tuning and introduced the $M$ factor. Sadleir et al. [6] saw clear structures in the critical current and baseline as function of perpendicular magnetic field which point towards weak-link behavior between the low $T_{c}$ MoAu TES and high $T_{c}$ leads. Ishisaki et al. [7] investigated the influence of magnetic field on TiAu X-ray TES detectors and have effectively shielded the TES for the magnetic field by adding a superconducting layer underneath the TES.

\section{Bolometer and Set-up}

For this experiment a well characterized Ti/Au bolometer is used with properties close to the specification of the final pixel design for the SAFARI instrument, Table 1 $[8,9]$. The bolometer is of the straight leg design; see Fig. 1, which has legs from the island to the substrate in an " $\mathrm{X}$ "-like geometry.

The measurements were conducted in an Oxford Instruments wet dilution refrigerator with a base temperature of $16 \mathrm{mK}$. The device was mounted inside a light-tight box to avoid optical loading from warmer parts. This box was mounted on the sample stage and surrounded by an aluminum shield to expel any remnant magnetic field. Lastly, the complete cryostat was surrounded by a series of two $\mu$-metal shields to expel the earth's magnetic field.

The TES was voltage biased and the current was read out using an 8-turn NIST SQUID located inside a $\mathrm{Nb}$ shield mounted on the sample space in the Al can. The 
Fig. 2 Bias circuit of a TES

Table 1 TES parameters

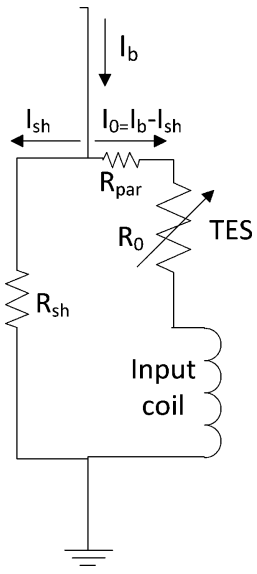

\begin{tabular}{ll}
\hline TES material & $\mathrm{Ti} / \mathrm{Au}$ \\
TES Thickness & $16 / 85 \mathrm{~nm}$ \\
TES Size & $50 \times 50 \mathrm{~mm}^{2}$ \\
Island/leg material & $\mathrm{Si}_{3} \mathrm{~N}_{4}$ \\
Island/leg thickness & $0.5 \mathrm{~nm}$ \\
Island size & $160 \times 160 \mu \mathrm{m}^{2}$ \\
Leg length & $400 \mu \mathrm{m}$ \\
$T_{C}$ & $85 \mathrm{mK}$ \\
$G$ & $0.38 \mathrm{pW} / \mathrm{K}$ \\
$R_{n}$ & $103 \mathrm{~m} \Omega$ \\
$N E P_{\text {meas }}$ (Measures Noise Equivalent Power) & $5 \times 10^{-19} \mathrm{~W} / \sqrt{ } \mathrm{Hz}$ \\
$P_{\text {sat }}$ (Saturation Power) & $12 \mathrm{fW}$ \\
\hline
\end{tabular}

output of the SQUID was connected to an ADC card for recording. The bias for the SQUID channel and the TES, as well as for the coils providing the applied magnetic fields was provided by Magnicon electronics at room temperature. These electronics were situated in an electrically filtered closed metal box on the cryostat, to shield against EMI.

The magnetic fields for the experiment were provided by two coils. The perpendicular field was produced using a circular coil with 220 turns. The coil was attached to the back of the light-tight box and produced a field of $71.5 \mathrm{G} / \mathrm{A}$ at the location of the TES. The coil used to produce the parallel field was a 15-turn coil which is positioned around the light tight box. It produced a field of $6 \mathrm{G} / \mathrm{A}$ at the location of the TES.

Figure 2 shows the basic bias circuit used to voltage bias a bolometer. This bias circuit does not supply a truly constant voltage bias but a constant current bias, $I_{b}$, through a small shunt resistor, $R_{s h}=4.9 \mathrm{~m} \Omega$. It is evident that when the resistance of the TES changes the current $I_{0}$ through the TES is also affected. Because $I_{b}$ is constant this will produce a change in the current through the shunt resistor affecting the voltage bias across the TES. 

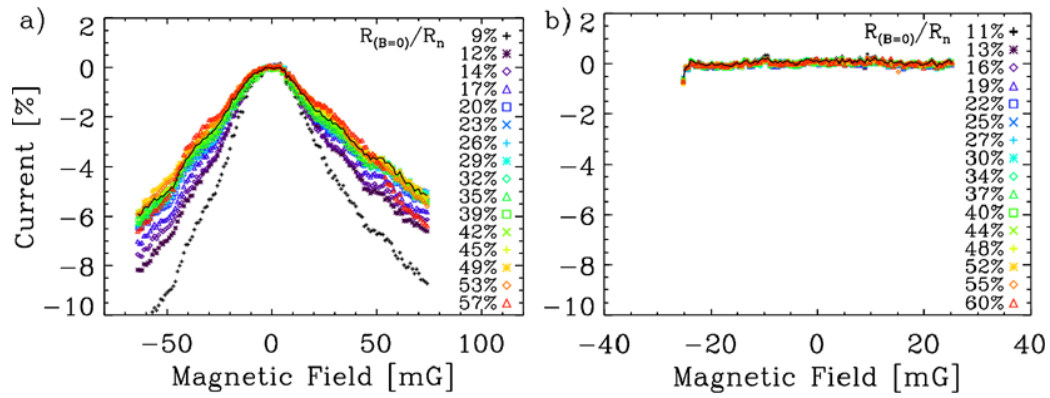

Fig. 3 (Color online) Normalized change in baseline TES current as function of applied magnetic field strength (a) perpendicular or (b) parallel to the TES. Measurements were performed at different bias points of the TES (shown as $\left.R / R_{n}\right)$. The change in baseline at the standard operating points $\left(R=0.3 R_{n}\right)$ is plotted as a solid line

\section{Baseline}

The baseline of the TES is defined as the current bias through the TES without any photon signal. A change in this current can be mistaken as a photon signal. For this reason the effect of the magnetic field on the baseline needs to be well understood. Below, we describe our results to understand this baseline as a function of applied magnetic field. First, we recorded a series of IV curves taken with known magnetic fields either perpendicular of parallel to the TES. We determined the TES baseline response by measuring the current present in the TES at a fixed bias current.

Figure 3 shows how the TES baseline current changes (relative to the baseline current for $B=0 \mathrm{mG}$ ) as a function of applied magnetic field either (a) perpendicular of (b) parallel to the TES. The magnetic fields strengths are calculated using the geometry of the coil and position of TES relative to the coil. The magnetic field values are corrected for the measured ambient field present in the set-up. This ambient field varies with location on the chip but is constant between cool downs. The uncertainty of this measurement is $0.1 \%$ which was determined by taking multiple IV curves and determining the spread in baseline. This uncertainty is mainly caused by the instability of the operating temperature.

In the range of $\pm 50 \mathrm{mG}$ for the perpendicular field the baseline reduces from zero field outwards due to suppression of superconductivity by the magnetic field. The change is $5 \%$ at $\pm 50 \mathrm{mG}$ for $R=0.3 R_{n}$. Also a structure with a period of $\pm 30 \mathrm{mG}$ is present. The parallel field has little to no effect on the baseline of the TES in the range $\pm 20 \mathrm{mG}$.

We also determined $d I / d B$ for the TES to get more insight on the magnetic field effects. The results are shown in Fig. 4. Most notably, for the first $15 \mathrm{mG}$ of the perpendicular field the variation of baseline rises to $0.7 \mathrm{nA} / \mathrm{mG}$, Fig. 4a). Beyond $15 \mathrm{mG}$ the variation in baseline fluctuates around this value. The effect of the parallel field is still negligible, Fig. 4b). 

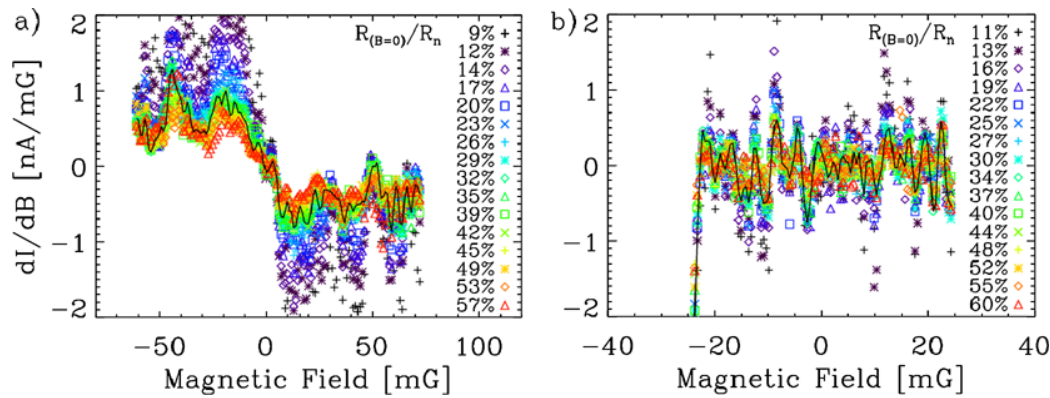

Fig. 4 (Color online) $d I / d B$ as a function of the (a) perpendicular and (b) parallel field, both plotted to the same scale for comparison. The $d I / d B$ at the operating point $\left(R=0.3 R_{n}\right)$ is plotted as a solid line
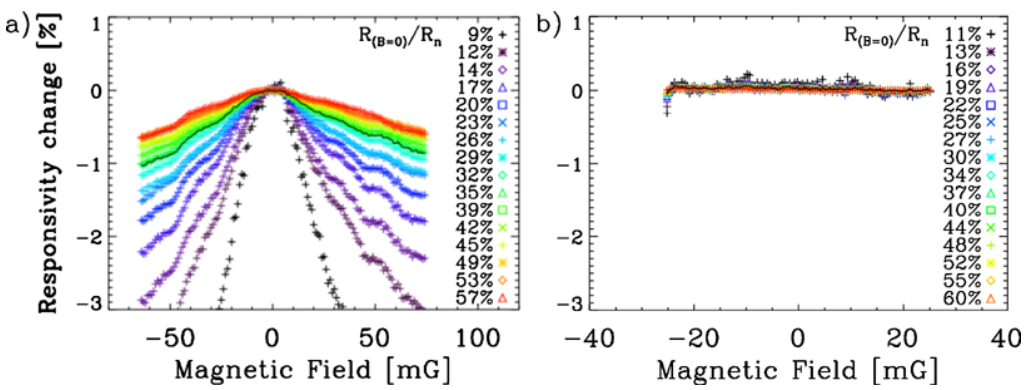

Fig. 5 (Color online) Change in responsivity at zero frequency as a function of (a) perpendicular and (b) parallel magnetic field. The change in $s(0)$ at the operating point $\left(R=0.3 R_{n}\right)$ is plotted as a solid line

\section{Responsivity}

A change in the responsivity will produce an incorrect interpretation of the signal measured by the TES. It is assumed that within this bandwidth of the SAFARI instrument the responsivity is constant with respect to the frequency. In this case a simplification can be used by only determining the responsivity at zero frequency given by [4]:

$$
s_{I, T E S}(0)=-\frac{1}{I_{0}\left(R_{0}-\left(R_{s h}+R_{p a r}\right)\right)}
$$

where $I_{0}$ the current through the TES, $R_{0}$ the resistance of the TES, $R_{p a r}$ the parasitic resistance and $R_{s h}$ the shunt resistance, see Fig. 2. By using the IV curve and (1) the responsivity can be obtained for different magnetic field throughout the transition. The results are shown in Fig. 5. The shape of the curve of responsivity versus perpendicular field is similar to the change in baseline, showing the same fluctuations, but smaller (Fig. 5a). At the operating point $\left(R=0.3 R_{n}\right)$ the responsivity changes $1 \%$ at $\pm 50 \mathrm{mG}$. The effect of the parallel field is again within the noise (Fig. 5b). 


\section{Discussion and Conclusion}

Our data for the baseline and responsivity of a TiAu TES with Ta absorber show a broad peak as a function of perpendicular magnetic field. We did not observe the fine structure effects seen by other groups [6]. Fluctuations are present, clearly seen in the $d I / d B$, but they are away from the main peak and will not affect device performance. The origin of these fluctuations is still under discussion. A Fraunhoffer pattern caused by weak link behaviour, as seen by Sadleir et al. [6], would have a period of $\sim 8 \mathrm{mG}$ in our measurement [10]. We observed a period of $30 \mathrm{mG}$ which can indicate that the TES is only partly affected. Furthermore, measurements at higher bath temperatures (not presented in the current paper) did not show a change in the fluctuations as what were observed by Sadleir et al.

The bandwidth of the bolometers of SAFARI will be 40 to $100 \mathrm{~Hz}$, which corresponds roughly to half the required detector speed. It is likely that changes in the speed of the device, e.g. due to the magnetic field, will affect the responsivity within this bandwidth. This will be investigated in more detail.

The results for the perpendicular field show that the TES bolometer can be used within field strength of $\pm 10 \mathrm{mG}$. The effect of the parallel field is smaller than the effect of the perpendicular field, as expected, due to the cross-section exposed to this field. However, our results also show that the effect of the parallel field is only a factor of five smaller than the effect of the perpendicular field. This difference of the factor of five seems to be smaller than one could expect. In order to possibly relax the constraints of shielding in the parallel direction, one would like to confirm this hypothesis. This can be achieved by improving the temperature stability in order to reduce the measurement uncertainty.

\section{References}

1. H. Kaneda, T. Nakagawa, T. Onaka, T. Matsumoto, H. Murakami, K. Enya, H. Kataza, H. Matsuhara, Y.Y. Yui, Proc. SPIE 5487, 991-1000 (2004)

2. B. Swinyard, Proc. SPIE 6265, 62650L (2006)

3. M.A. Lindeman, S. Bandler, R.P. Brekosky, J.A. Chervenak, E. Figueroa-Feliciano, F.M. Finkbeiner, M.J. Li, C.A. Kilbourne, Rev. Sci. Instrum. 75, 1283 (2004)

4. K.D. Irwin, G.C. Hilton, in Topics in Applied Physics: “Cryogenic Particle Detection”, ed. by C. Enss (Springer, Berlin, 2005)

5. J.N. Ullom, W.B. Doriese, G.C. Hilton, J.A. Beall, S. Deiker, W.D. Duncan, L. Ferreira, K.D. Irwin, C.D. Reintsema, L.R. Vale, Appl. Phys. Lett. 84, 4206 (2004)

6. J.E. Sadleir, S.J. Smith, S.R. Bandler, J.A. Chervenak, J.R. Clem, Phys. Rev. Lett. 104, 047003 (2010)

7. Y. Ishisaki, H. Kurabayashi, A. Hoshino, T. Ohashi, T. Yoshino, T. Hagihara, K. Mitsuda, K. Tanaka, J. Low Temp. Phys. 151, 131 (2004)

8. P. Khosropanah, R.A. Hijmering, M. Ridder, M. Lindeman, L. Gottardi, M. Bruijn, J. van der Kuur, P. de Korte, J.-R. Gao, H. Hoevers, J. Low Temp. Phys. (2012). doi:10.1007/s10909-012-0550-6

9. M. Lindeman, P. Khosropanah, R.A. Hijmering, M. Ridder, L. Gottardi, M. Bruijn, J. van der Kuur, P. de Korte, J.-R. Gao, H. Hoevers, J. Low Temp. Phys. (2012). doi:10.1007/s10909-012-0572-0

10. R.L. Peterson, Cryogenics 31, 2088-2091 (1993) 